\title{
PROPER MULTIPLE INTEGRALS OVER ITERABLE FIELDS
}

\author{
BY \\ ERNEST B. LYTLE* \\ Introduction.
}

The theory of multiple integrals has generally been confined to functions having metric (defined later in $\S 1$ ) domains of definition. In his book $\dagger$ and in an article on Improper Multiple Integrals $\ddagger$ Pierpon' has given a definition of integrals applicable to non-metric domains.

Using this Pierpont definition of an integral I have found a new class of fields for which the fundamental relation

$$
\int_{\mathscr{X}} f \leqq \int_{\mathbb{Y}} \int_{\mathbb{S}} f \leqq \int_{\mathbb{B}} \int_{\mathfrak{S}}^{\bar{S}} f \leqq \int_{\mathscr{X}} f
$$

holds. This general class of fields, which I have called iterable, includes all metric fields and a large class of non-metric fields.

For simplicity the results are stated throughout the paper for functions of two independent variables; but the method of treatment is general and the extension to the case of any number of independent variables is thought to be obvious. The notation used by Pierpont has been followed, and the reader is referred for further explanation to his Lectures.

\section{§1. Preliminary definitions.}

Let $\mathfrak{A}=\{x, y\}$ be a two-dimensional point aggregate lying within a square. Effect a rectangular division of the plane of norm $\delta$. Let

$$
\delta_{1}^{\prime}, \delta_{2}^{\prime}, \delta_{3}^{\prime}, \ldots
$$

be those rectangles containing at least one point of $\mathfrak{A}$. Let

$$
\delta_{1}^{\prime \prime}, \delta_{2}^{\prime \prime}, \delta_{3}^{\prime \prime}, \ldots
$$

denote those rectangles all of whose points belong to $\mathfrak{A}$. Then

$$
\lim _{\delta=0} \Sigma \delta_{i}^{\prime}=\overline{\mathfrak{A}}
$$

* Presented to the Society (New York), April 25, 1908.

$\dagger$ Lectures on the Theory of Functions of Real Variables, vol. 1 (1905). I shall refer to this as Lectures.

† These Transactions, vol. 7 (1906). Referred to later under the title Improper Integrals. 
is defined to be the outer (upper) content of $\mathfrak{A}$, and

$$
\lim _{\delta=0} \Sigma \delta_{i}^{\prime \prime}=\underline{\mathfrak{X}}
$$

is the inner (lower) content of $\mathfrak{A}$. It has been shown (Lectures, p. 513) that these limits always exist and are finite. When

$$
\overline{\mathfrak{U}}=\underline{\mathfrak{A}}
$$

their common value is called the content of $\mathfrak{A}$, and $\mathfrak{A}$ is said to be a metric aggregate.

Let $\mathfrak{A}=\{x, y\}$ be a limited point aggregate. Let $f(x, y)$ be a limited single-valued function defined over $\mathfrak{A}$. Effect a rectangular division $D$ of the plane of norm $\delta$. Let

$$
\delta_{1}^{\prime}, \delta_{2}^{\prime}, \delta_{3}^{\prime}, \cdots,
$$

be those rectangles of $D$ which contain points of $\mathfrak{A}$. Let $M_{i}, m_{i}$ be the maximum and minimum respectively of $f(x, y)$ in the rectangle $\delta_{i}^{\prime}$. Put

$$
\bar{S}_{D}=\Sigma M_{i} \delta_{i}^{\prime}, \quad \underline{S}_{D}=\Sigma m_{i} \delta_{i}^{\prime} .
$$

Then

$$
\lim _{\delta=0} \bar{S}_{D}=\bar{\int}_{\mathfrak{x}} f
$$

is defined to be the outer upper integral of $f(x, y)$ over the field $\mathfrak{A}$, and

$$
\lim _{\delta=0} \underline{S}_{D}=\int_{\mathscr{x}} f
$$

is the outer lower integral of $f(x, y)$ over $\mathfrak{A}$. These limits always exist and are finite. (See Lectures, p. 510.)

When the integrals (2) and (3) are equal we denote their common value by

$$
\int_{\mathfrak{x}} f
$$

and say that $f$ is integrable over $\mathfrak{A}$.

It is important to note that the relations

$$
\bar{\int}_{\mathscr{x}} f=\min \bar{S}_{D}, \quad \int_{\mathscr{x}} f=\max \underline{S}_{D}
$$

which hold for Riemann integrals are not always true for outer integrals. (See Lectures, p. 508.)

Let $\mathfrak{A}$ be a limited plane point set. Let $\mathfrak{C}$ be the points of $\mathfrak{A}$ lying on a line parallel to the $y$-axis. Let $\mathfrak{B}$ be the projection of $\mathfrak{A}$ on the $x$-axis. Then when

$$
\int_{\mathfrak{B}} \overline{\mathfrak{E}}=\overline{\mathfrak{A}}
$$

we define $\mathfrak{A}$ to be iterable relatively to $x$. 
If $C$ is a linear section of $\mathfrak{A}$ parallel to the $x$-axis and $B$ is the projection of $\mathfrak{A}$ on the $y$-axis, then when

$$
\int_{B} \bar{C}=\overline{\mathfrak{A}}
$$

$\mathfrak{A}$ is iterable relatively to $y$.

The following examples show that both iterable and non-iterable aggregates exist.

Example 1. Let $\mathfrak{A}=\{x, y\}$ where $x$ takes the rational values in a unit interval and $y$ takes all real values in a unit interval. Then $\mathfrak{A}$ is non-metric, for

$$
\underline{\mathfrak{A}}=0, \quad \overline{\mathfrak{A}}=1 .
$$

But $\mathfrak{A}$ is iterable relative to both $x$ and $y$, for

$$
\int_{\mathfrak{B}} \overline{\mathfrak{C}}=\int_{B} \bar{C}=1=\overline{\mathfrak{A}} \text {. }
$$

Example 2. Let $\mathfrak{A}=\{x, y\}$ be the points of a unit square, specified for rational $x$ by $0 \leqq y \leqq \frac{1}{2}$, and for irrational $x$ by $\frac{1}{2} \leqq y \leqq 1$. Here $\mathfrak{A}$ is non-metric, for

$$
\underline{\mathfrak{A}}=\mathbf{0}, \quad \overline{\mathfrak{A}}=\mathbf{1} .
$$

Also $\mathfrak{A}$ is iterable relatively to $y$, for

$$
\int_{B} \bar{C}=1=\overline{\mathfrak{A}}
$$

but not iterable relatively to $x$, for

$$
\int_{\mathfrak{B}} \overline{\mathfrak{E}}=\frac{1}{2} \neq \overline{\mathfrak{A}}
$$

By similar methods fields may readily be constructed which are iterable neither relatively to $x$ nor relatively to $y$.

Let

and for each $x$ let

$$
\mathfrak{A}=\mathfrak{A}_{1}+\mathfrak{A}_{2},
$$

$$
\overline{\mathfrak{E}}=\overline{\mathfrak{E}}_{1}=\overline{\mathfrak{E}}_{2} \text {. }
$$

Then we say that $\mathfrak{A}_{1}$ and $\mathfrak{A}_{2}$ are thoroughly mixed partials of $\mathfrak{A}$.

The notation $\mathfrak{A}=\mathfrak{B} \cdot \mathfrak{C}$ is used to specify that $\mathfrak{B}$ is the projection of $\mathfrak{A}$ on the $x$-axis and $\mathfrak{C}$ is a linear section of $\mathfrak{A}$ parallel to the $y$-axis.

\section{§2. Properties of iterable fields.}

TheOREM 1. Every metric aggregate is iterable relatively to both $x$ and $y$. It has been shown (see Lectures, p. 538) that, for a function $f(x, y)$ limited 
in the metric field $\mathfrak{A}$,

$$
\begin{aligned}
& \int_{\mathscr{M}} f \leqq \underline{\int} d x \bar{\int} f d y \leqq \bar{\int} d x \bar{\int} f d y \leqq \overline{\int_{\mathfrak{x}}} \dot{ }, \\
& \int_{\mathscr{x}} f \leqq \int d y \bar{\int} f d x \leqq \bar{\int} d y \bar{\int} f d x \leqq \int_{\mathbb{N}} f .
\end{aligned}
$$

In these relations put $f(x, y)=1$ and they become

Hence

$$
\overline{\mathfrak{A}} \leqq \int_{B} \bar{C} \leqq \int_{B} \bar{C} \leqq \overline{\mathfrak{A}}, \quad \overline{\mathfrak{A}} \leqq \int_{\mathscr{B}} \overline{\mathfrak{N}} \leqq \overline{\int_{\mathfrak{A}}} \overline{\mathfrak{E}} \leqq \overline{\mathfrak{A}} .
$$

$$
\int_{\mathfrak{y}} \overline{\mathfrak{E}}=\int_{B} \bar{C}=\overline{\mathfrak{A}}
$$

that is, $\mathfrak{A}$ is iterable relatively to $x$ and relatively to $y$.

Lemma. Let $\mathfrak{A}$ be iterable relatively to $x$. Then if $\delta=\alpha \beta$ is a cell of any rectangular division $D$, we have

$$
\lim _{\delta=0} \Sigma(\alpha \Sigma \beta)=\lim _{\alpha=0} \Sigma\left(\alpha \lim _{\beta=0} \Sigma \beta\right) .
$$

For each $x$ the inequality

will hold. Hence

$$
\lim _{\beta=0} \Sigma \beta \leqq \Sigma_{\beta}
$$

But

$$
\Sigma\left(\alpha \lim _{\beta=0} \Sigma \beta\right) \leqq \Sigma(\alpha \Sigma \beta) \leqq \Sigma \delta \text {. }
$$

$$
\begin{gathered}
\lim _{\delta=0} \Sigma\left(\alpha \lim _{\beta=0} \Sigma \beta\right)=\lim _{\alpha=0} \Sigma\left(\alpha \lim _{\beta=0} \Sigma \beta\right)=\int_{\mathscr{Y}} \overline{\mathfrak{E}}, \\
\lim _{\delta=0} \Sigma \delta=\overline{\mathfrak{A}} .
\end{gathered}
$$

Since $\mathfrak{A}$ is iterable relatively to $x$

$$
\int_{\mathfrak{B}} \overline{\mathfrak{E}}=\overline{\mathfrak{A}}
$$

and our theorem follows.

This lemma enables us to evaluate an iterated limit by a simpler single limit. A similar lemma is true for a field iterable relatively to $y$.

Theorem 2. Every partial unmixed aggregate of an iterable aggregate is iterable.

Let

$$
\mathfrak{A}=\mathfrak{A}_{1}+\mathfrak{A}_{2},
$$

where $\mathfrak{A}$ is iterable and $\mathfrak{X}_{1}$ and $\mathfrak{A}_{2}$ are unmixed.*

* See Lectures, \& 711, p. 519. 
Then for each $x$

and for every $x$-interval

$$
\overline{\mathfrak{E}} \leqq \overline{\mathfrak{E}}_{1}+\overline{\mathbb{E}_{2}},
$$

$$
\min \overline{\mathfrak{C}} \leqq \min \overline{\mathfrak{E}}_{1}+\max \overline{\mathfrak{E}}_{2} .
$$

Hence by integration over $\mathfrak{B}$,

But

$$
\int \overline{\mathfrak{E}} \leqq \int \overline{\mathfrak{E}}_{1}+\bar{\int}_{\overline{\mathfrak{E}}} .
$$

Hence

$$
\int \overline{\mathfrak{E}}_{1} \leqq \overline{\mathfrak{A}}_{1}, \quad \int \overline{\mathfrak{C}}_{\mathfrak{\mathfrak { E }}_{2}} \leqq \overline{\mathfrak{A}}_{2} .
$$

$$
\int \overline{\mathfrak{E}} \leqq \int \overline{\mathfrak{E}}_{1}+\int \overline{\mathfrak{E}} \overline{\mathfrak{E}}_{2} \leqq \overline{\mathfrak{A}}_{1}+\overline{\mathfrak{A}}_{2} .
$$

Since $\mathfrak{A}$ is iterable by hypothesis

$$
\int \overline{\mathfrak{E}}=\overline{\mathfrak{A}}
$$

and since $\mathfrak{A}_{1}$ and $\mathfrak{A}_{2}$ are unmixed

Therefore by (1)

$$
\overline{\mathfrak{A}}=\overline{\mathfrak{A}}_{1}+\overline{\mathfrak{A}}_{2} .
$$

But

$$
\int \overline{\mathfrak{E}}_{1}+\bar{\int} \overline{\mathfrak{E}}_{2}=\overline{\mathfrak{A}}_{1}+\overline{\mathfrak{A}}_{2},
$$

or

hence

$$
\left(\overline{\mathfrak{A}}_{1}-\underline{\int} \mathfrak{C}_{1}\right)+\left(\overline{\mathfrak{A}}_{2}-\bar{\int} \mathfrak{E}_{2}\right)=0 .
$$

$$
\overline{\mathfrak{A}}_{1}-\int \overline{\mathfrak{E}}_{1} \geqq 0, \quad \overline{\mathfrak{A}}_{2}-\bar{\int} \overline{\mathfrak{E}}_{2} \geqq 0,
$$

\section{Similarly}

(3)

$$
\overline{\mathfrak{A}}_{1}=\int \overline{\mathfrak{E}}_{1}, \quad \overline{\mathfrak{A}}_{2}=\bar{\int}_{\overline{\mathfrak{E}}} .
$$

from which follows

$$
\int \overline{\mathfrak{E}} \leqq \bar{\int} \overline{\mathfrak{E}}_{1}+\int \overline{\mathfrak{E}}_{2} \leqq \overline{\mathfrak{A}}_{1}+\overline{\mathfrak{A}}_{2}=\overline{\mathfrak{A}},
$$

$$
\overline{\mathfrak{A}}_{1}=\bar{\int} \overline{\mathfrak{E}}_{1}, \quad \int \underline{\mathfrak{A}_{2}}=\overline{\mathfrak{E}}_{2} .
$$

By (2) and (4), $\mathfrak{A}_{1}$ and $\mathfrak{A}_{2}$ are each iterable. 
CoROLlary. If a discrete aggregate be subtracted from an iterable aggregate, then the remaining aggregate is iterable.

Let

$$
\mathfrak{A}=\mathfrak{A}_{1}+\mathfrak{A}_{2},
$$

where $\mathfrak{A}$ is iterable and $\mathfrak{A}_{2}$ is discrete. Since $\mathfrak{A}_{2}$ is discrete $\mathfrak{A}_{1}$ and $\mathfrak{A}_{2}$ are unmixed. Therefore $\mathfrak{A}_{1}$ is iterable by the preceding theorem.

\section{§3. Proper Integrals.}

A proper integral is one in which both the function and its field of integration are limited.

TheOREM 3.* Let $f(x, y) \geqq 0$ be limited over the limited field $\mathfrak{A}$. Then

$$
\overline{\int_{\mathfrak{B}}} \overline{\int_{\mathfrak{E}}} f \leqq \overline{\int_{\mathfrak{R}}} f .
$$

If the sign of $f$ is changed, the theorem is converted into the following:

Theorem 4. Let $f(x, y) \leqq 0$ be limited over the limited field $\mathfrak{A}$. Then

$$
\int_{\mathfrak{x}} f \leqq \int_{\mathfrak{B}} \int_{\mathfrak{E}}^{\mathfrak{e}} f
$$

ThLorem 5. Let $f(x, y)$ and $g(x, y)$ each be limited over the limited field $\mathfrak{A}$. Let them be equal except at a discrete set of points. Then

$$
\begin{gathered}
\bar{\int}_{\mathfrak{x}} f=\bar{\int}_{\mathfrak{A}} g, \\
\overline{\int_{\mathfrak{B}}} \overline{\int_{\mathfrak{E}}} f=\overline{\int_{\mathfrak{B}}} \overline{\int_{\mathfrak{S}}} g .
\end{gathered}
$$

Let $\mathfrak{A}=\mathfrak{A}_{1}+\mathfrak{A}_{2}$, where $\mathfrak{A}_{2}$ is discrete. Let $f=g$ over $\mathfrak{A}_{1}$. Let $D$ be a rectangular division of the plane of norm $\delta=\alpha \cdot \beta$. Let

$$
\delta_{11}, \delta_{12}, \delta_{13}, \ldots
$$

be those rectangles which contain no points of $\mathfrak{A}_{2}$. Let contain points of $\mathfrak{U}_{2}$. Then

$$
\delta_{21}, \delta_{22}, \delta_{23}, \ldots
$$

$$
\begin{aligned}
& \Sigma M_{i} \delta_{i}=\Sigma M_{1 i} \delta_{1 i}+\Sigma M_{2 i} \delta_{2 i}, \\
& \Sigma N_{i} \delta_{i}=\Sigma N_{1 i} \delta_{1 i}+\Sigma N_{2 i} \delta_{2 i},
\end{aligned}
$$

* Restated for later use. For proof see RICHARDson, these Transactions, vol. 9 (1908), p. 347. 
where $M_{i}$ and $N_{i}$ are the maxima of $f$ and $g$ respectively in $\delta_{i}$. Subtracting we get

$$
\Sigma M_{i} \delta_{i}-\Sigma N_{i} \delta_{i}=\Sigma M_{2 i} \delta_{2 i}-\Sigma N_{2 i} \delta_{2 i}
$$

since $M_{1 i}=N_{1 i}$. On passing to the limit as $\delta \doteq 0$, this becomes

$$
\bar{\int}_{\mathfrak{x}} f-\bar{\int}_{\mathfrak{x}} g=0
$$

for each limit in the second member is zero because $\mathfrak{A}_{2}$ is discrete and both $f$ and $g$ are limited. This gives our relation $(a)$.

From $(a)$ and theorem 10 below it follows that

$$
\overline{\int_{\mathfrak{G}}} g=\bar{\int}_{\mathfrak{G}} f
$$

at all points of $\mathfrak{B}$ except a discrete set. Relation $(b)$ then follows by application of relation $(a)$ to the set $\mathfrak{B}$.

Theorem 6. Let $f(x, y)$ be limited over a limited field $\mathfrak{A}$ and negative only at a discrete set of points $\Delta$. Then

$$
\overline{\int_{\mathfrak{R}}} \overline{\int_{\mathfrak{E}}} f \leqq \bar{\int}_{\mathfrak{R}} f
$$

Let $g(x, y)=f(x, y)$ at all points of $\mathfrak{A}$ except at $\Delta$ where $g=0$. Then $g \geqq 0$, and by theorem 3

But by the preceding theorem

$$
\overline{\int_{\mathfrak{B}}} \overline{\int_{\mathfrak{E}}} g \leqq \overline{\int_{\mathfrak{X}}} g .
$$

$$
\overline{\int_{\mathfrak{Q}}} g=\overline{\int_{\mathfrak{A}}} f, \quad \overline{\int_{\mathfrak{B}}} \overline{\int_{\mathfrak{G}}} g=\bar{\int}_{\mathfrak{B}} \bar{\int}_{\mathfrak{E}} f,
$$

and substituting these in the above relation we have our theorem.

Theorem 7. Let $f(x, y)$ be limited over a limited field $\mathfrak{A}$ and positive only at a discrete set of points. Then

$$
\int_{\mathscr{T}} f \leqq \int_{\mathscr{B}} \int_{\mathbb{S}} f
$$

Theorem 8. Let $f(x, y)$ be limited over the limited field $\mathfrak{A}=\mathfrak{A}_{1}+\mathfrak{A}_{2}$. For each $x$ let $\overrightarrow{\mathfrak{E}}=\overline{\mathfrak{E}}_{1}=\overline{\mathfrak{E}}_{2}$. Let $f \geqq 0$ over $\mathfrak{A}_{1}$ and $f \leqq 0$ over $\mathfrak{A}_{2}$. Then

$$
\int_{\mathscr{X}} f \leqq \int_{\mathfrak{B}} \int_{\mathfrak{G}} f \leqq \int_{\mathbb{B}} \int_{\mathfrak{G}} f \leqq \int_{\mathscr{X}} f .
$$

This inequality will appear frequently in the succeeding pages and we shall refer to it as relation $(A)$. 
Since $\overline{\mathbb{E}}=\overline{\mathbb{E}}_{1}=\overline{\mathbb{E}}_{2}$, the positive and negative values of the function are each everywhere dense in $\mathfrak{A}$. Hence every rectangle containing a point of $\mathfrak{A}$ also contains a point of $\mathfrak{A}_{1}$, and

$$
\int_{\mathfrak{x}} f=\lim \Sigma_{\mathfrak{x}} M_{i} \delta_{i}=\lim \Sigma_{\mathfrak{x _ { 1 }}} M_{i} \delta_{i}=\int_{\mathfrak{x}_{1}} f .
$$

Similarly for each $x$ we have

$$
\overline{\int_{\mathfrak{G}}} f=\int_{\mathfrak{G}_{1}} f .
$$

But the projections of $\mathfrak{U}$ and $\mathfrak{A}_{1}$ on the $x$-axis are equal, hence

$$
\overline{\int_{B}} \overline{\int_{\mathfrak{E}}} f=\overline{\int_{\mathfrak{B}}} \bar{\int}_{\mathfrak{E}_{1}} f .
$$

Since $f \geqq 0$ over $\mathfrak{A}_{1}$, we have by theorem 3 ,

$$
\overline{\int_{\mathfrak{B}}} \int_{\mathfrak{E}_{1}}^{\overline{1}} f \leqq \int_{\mathfrak{x}_{1}}^{\bar{C}} f
$$

and the equals of both members give

$$
\overline{\int_{\mathfrak{D}}} \overline{\int_{\mathfrak{E}}} f \leqq \overline{\int_{\mathfrak{x}}} f
$$

In the same way, using theorem 4 and $\mathfrak{A}_{2}$, we get

But we always have

$$
\int_{\mathbb{A}} f \leqq \int_{\mathbb{Z}} \int_{\mathbb{S}} f
$$

$$
\int_{\mathfrak{B}} \int_{\mathfrak{G}} f \leqq \overline{\int_{\mathfrak{B}}} \int_{\mathfrak{E}}^{\bar{C}} f .
$$

Combining (1), (2) and (3) we get relation $(A)$, which was to be proved.

Examples of thoroughly mixed sets $\mathfrak{A}_{1}$ and $\mathfrak{A}_{2}$ are well known; for example, in $\mathfrak{A}_{1}$ let $x$ be real and $y$ be rational, and in $\mathfrak{A}_{2}$ let $x$ be real and $y$ be irrational.

Theorem 9. Let $f(x, y)$ be limited over the limited iterable field $\mathfrak{A}$. Then the existence of the multiple integral $\int_{\mathfrak{x}} f$ and the iterated integral $\int_{\mathfrak{B}} \int_{\mathfrak{E}} f$ is sufficient for their equality.

Let $|f| \leqq F$. Then $f+F \geqq 0$, and by theorem 3

$$
\overline{\int_{\mathscr{B}}} \overline{\int_{\mathfrak{E}}}\left(f+F^{\prime}\right) \leqq \int_{\mathscr{T}}^{\bar{x}}(f+F) .
$$


But

$\int_{\mathfrak{B}} \int_{\mathfrak{E}} f+F \int_{\mathfrak{B}} \overline{\mathfrak{E}}=\int_{\mathfrak{B}} \int_{\mathfrak{E}}(f+F) \leqq \int_{\mathfrak{B}} \int_{\mathfrak{E}}\left(f+F^{\prime}\right) \leqq \int_{\mathfrak{A}}\left(f+F^{\prime}\right)$

Hence

$$
=\int_{\mathfrak{x}} f+F \overline{\mathfrak{U}}=\int_{\mathfrak{x}} f+F \overline{\mathfrak{U}} \text {. }
$$

$$
\int_{\mathfrak{g}} \int_{\mathfrak{E}} f \leqq \int_{\mathfrak{x}} f+F\left(\overline{\mathfrak{A}}-\int_{\mathfrak{B}} \overline{\mathfrak{E}}\right) .
$$

Since $f-F \leqq 0$, we get in a similar way by theorem 4 ,

$$
\int_{\mathfrak{x}} f-F \overline{\mathfrak{A}}=\int_{\mathfrak{x}}\left(f-F^{\prime}\right) \leqq \int_{\mathfrak{g}} \int_{\mathfrak{E}}\left(f-F^{\prime}\right)=\int_{\mathfrak{B}} \int_{\mathfrak{E}} f-F \int_{\mathfrak{B}} \overline{\mathfrak{E}},
$$

and therefore

But since $\mathfrak{A}$ is iterable

$$
-F\left(\overline{\mathfrak{A}}-\int_{\mathfrak{B}} \overline{\mathfrak{E}}\right)+\int_{\mathfrak{x}} f \leq \int_{\mathfrak{B}} \int_{\mathfrak{E}} f
$$

and this with (1) and (2) gives

$$
\overline{\mathfrak{A}}-\int_{\mathfrak{B}} \overline{\mathfrak{E}}=0,
$$

$$
\int_{\mathfrak{x}} f=\int_{\mathfrak{B}} \int_{\mathfrak{B}} f
$$

We observe in this connection that the mere existence of the multiple and the iterated integrals is not sufficient in general for their equality. * The theorem just cited may not be true when the field is not iterable and the function limited, as is shown by the following example.

Example 3. Let $f(x, y)=1$ over the field $\mathfrak{A}=\{x, y\}$ where for rational $x, 0 \leqq y \leqq \frac{1}{2}$ and for irrational $x, \frac{1}{2} \leqq y \leqq 1$. Then

$$
\int_{\mathfrak{R}} f=1, \quad \int_{\mathfrak{B}} \int_{\mathfrak{E}} f=\frac{1}{2} \text {. }
$$

That is, both integrals exist but are unequal.

Theorem 10. Let $\mathfrak{A}=\mathfrak{B} \cdot \mathfrak{C}$ be discrete. Then the points $\mathfrak{B}_{\sigma}$ at which $\overline{\mathfrak{E}} \geqq \sigma$, where $\sigma$ denotes an arbitrary small positive number; form a discrete set. $\dagger$

Theorem 11. Let $f(x, y) \geqq 0$ be limited over the limited field $\mathfrak{A}$.

* Bulletin of the American Mathematical Society, November, 1906, p. 61.

†Cf. SchöNFLIES: Die Entwickelung der Lehre von den Punktmannigfaltigkeiten, Jahresbericht der deutwhen Mathematiker-Vereinigung, vol. 8 (1900), p. 96.

Trans. Am. Math. Soc. 3 
Let $\int_{\mathscr{8}} \overline{\mathfrak{E}}=\overline{\mathfrak{A}} . \quad$ Then

$$
\int_{\mathfrak{X}} f \leqq \int_{\mathfrak{B}} \int_{\mathfrak{G}} f \leqq \int_{\mathfrak{B}}^{\bar{S}} \bar{\int}_{\mathfrak{S}} f \leqq \int_{\mathfrak{A}} f .
$$

By theorem 3 we have

$$
\overline{\int_{\mathfrak{B}}} \overline{\int_{\mathfrak{E}}} f \leqq \overline{\int_{\mathfrak{x}}} f
$$

But $f-F \leqq 0$, hence by theorem 4 and Lectures, p. 535, we have

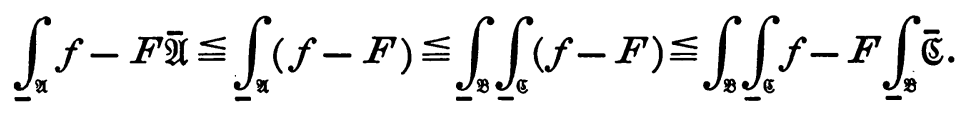

And since by hypothesis $\overline{\mathfrak{A}}=\int_{\mathscr{B}} \overline{\mathfrak{E}}$, this gives

$$
\int_{\mathscr{Q}} f \leqq \int_{\mathbb{B}} \int_{\mathscr{S}} f
$$

Relation $(A)$ then follows from (1) and (2).

Theorem 12. Let $f(x, y) \leqq 0$ be limited over the limited field $\mathfrak{A}$. Let $\int_{\mathscr{B}} \overline{\mathfrak{E}}=\overline{\mathfrak{A}} . \quad$ Then

$$
\int_{\mathscr{X}} f \leqq \int_{\mathscr{P}} \int_{\mathfrak{G}} f \leqq \int_{\mathfrak{P}} \bar{\int}_{\mathfrak{G}} f \leqq \int_{\mathscr{T}} f .
$$

Theorem 13. Let $\left.f^{\prime} \because, y\right)$ be limited over the limited field $\mathfrak{A}$. Let $\mathfrak{C}$ be an integrable functiun of $x$ and let $C$ be any constant. Then

$$
\begin{aligned}
& \int_{\mathfrak{x}}(f+C)=\int_{\mathfrak{x}} f+C \overline{\mathfrak{A}} . \\
& \overline{\int_{\mathfrak{B}}} \overline{\int_{\mathfrak{E}}}(f+C)=\overline{\int_{\mathfrak{B}}} \overline{\int_{\mathfrak{E}}} f+C \int_{\mathfrak{B}} \overline{\mathfrak{E}} .
\end{aligned}
$$

Relation (e) follows as an application of a corollary of RICHARDson's.* By an application of relation (e) we get relation $(f)$, thus

$$
\begin{aligned}
\overline{\int_{\mathscr{B}}} \overline{\int_{\mathfrak{E}}}(f+C) & =\overline{\int_{\mathfrak{B}}}\left[\overline{\int_{\mathfrak{E}}} f+C \overline{\mathfrak{E}}\right] \\
& =\int_{\mathscr{D}} \overline{\int_{\mathfrak{E}}} f+C \int_{\mathscr{E}} \overline{\mathfrak{E}}
\end{aligned}
$$

by hypothesis and Richardson's corollary.

* These Transactions, vol. 9 (1908), p. 350. 
We may obtain similar relations for lower integrals.

Theorem 14. Let $f(x, y)$ be limited over the limited field $\mathfrak{A}$ which is iterable relatively to $x$. Then

$$
\int_{\mathscr{X}} f \leqq \int_{\mathscr{D}} \int_{\mathscr{E}} f \leqq \bar{\int}_{\mathscr{S}} \bar{\int}_{\mathscr{B}} f \leqq \bar{\int}_{\mathscr{X}} f .
$$

Since $f+F \geqq 0$, by theorem 3

$$
\overline{\int_{\mathfrak{y}}} \int_{\mathfrak{E}}(f+F) \leqq \overline{\int_{\mathfrak{x}}}\left(f+F^{\prime}\right) .
$$

By theorem 13 we have

Therefore

$$
\begin{gathered}
\int_{\mathfrak{B}} \int_{\mathfrak{E}}(f+F)=\bar{\int}_{\mathfrak{B}} \int_{\mathfrak{E}} f+F \int_{\mathfrak{B}} \overline{\mathfrak{S}} \\
\overline{\int_{\mathfrak{X}}}(f+F)=\bar{\int}_{\mathfrak{X}} f+F \overline{\mathfrak{A}} .
\end{gathered}
$$

$$
\overline{\int_{\mathfrak{B}}} \overline{\int_{\mathfrak{E}}} f \leqq \overline{\int_{\mathfrak{a}}} f+F\left(\overline{\mathfrak{A}}-\int_{\mathfrak{B}} \overline{\mathfrak{E}}\right) .
$$

Again, since $f-F \leqq 0$, by theorem 4

$$
\underline{\int}_{\mathfrak{x}}\left(f-F^{\prime}\right) \leqq \int_{\mathfrak{B}} \int_{\mathfrak{G}}\left(f-F^{\prime}\right) .
$$

But by relations for lower integrals similar to those of theorem 13

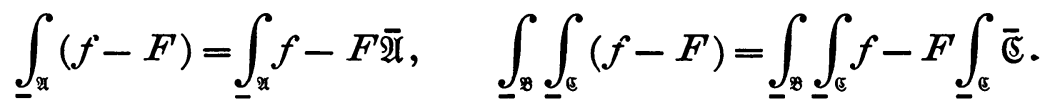

Therefore

$$
-F\left(\overline{\mathfrak{A}}-\int_{\mathscr{B}} \overline{\mathfrak{E}}\right)+\int_{\mathscr{X}} f \leqq \int_{\mathfrak{B}} \int_{\mathfrak{E}} f
$$

But $\mathfrak{A}$ being iterable, we have

$$
\overline{\mathfrak{A}}-\int_{\mathfrak{B}} \overline{\mathfrak{E}}=0 .
$$

Therefore relation $(A)$ follows at once from (1), (2) and (3).

Relation $(A)$ may be written

$$
\underline{\int} f d \mathfrak{A} \leqq \underline{\int} d x f d y \leqq \bar{\int} d x \bar{\int} f d y \leqq \bar{\int} f d \mathfrak{A} .
$$


If the field $\mathfrak{A}$ is iterable relatively to $y$, we obtain in a similar way

$$
\underline{\int} f d \mathfrak{A} \leqq \underline{\int} d y \underline{\int} f d x \leqq \bar{\int} d y \bar{\int} f d x \leqq \bar{\int} f d \mathfrak{A} .
$$

Corollary 1. Let $f(x, y)$ be integrable over any limited field which is iterable relatively to both $x$ and $y$. Let $f$ also be integrable over any section of $\mathfrak{A}$ parallel to either axis. Then

$$
\int f d \mathfrak{U}=\int d x \int f d y=\int d y \int f d x .
$$

This follows at once by putting

$$
\int_{\mathfrak{x}} f d \mathfrak{A}=\bar{\int} f d \mathfrak{A}=\int f d \mathfrak{A}
$$

in relations $(g)$ and $(h)$ above. It gives a very general condition for inversion in iterated integrals.

In conclusion, it may be shown that the equality

$$
\int_{\mathbb{B}} \int_{\mathfrak{G}} f=\int_{\mathbb{8}}^{\overline{0}} \int_{\mathfrak{G}} f
$$

is not sufficient for the existence of

$$
\int_{\mathfrak{B}} \int_{\mathfrak{G}} f
$$

For consider the following example. Let $\mathfrak{A}=\{x, y\}$, where $0 \leqq x=p / q \leqq 1$, $0 \leqq y \leqq 1 / q$. For rational $y$ let $f(x, y)=+1$ and for irrational $y$ let $f(x, y)=-1$. Then $\mathfrak{A}$ is discrete and $f$ is limited, bence

by theorem 14. But

$$
\int_{\mathfrak{A}} f=\int_{\mathfrak{B}} \int_{\mathfrak{E}} f=\int_{\mathscr{B}} \bar{\int}_{\mathfrak{E}} f=0
$$

$$
\int_{\mathbb{E}} f=-\frac{1}{q}, \quad \int_{\mathbb{E}} f=+\frac{1}{q}
$$

for each $x$ in $\mathfrak{B}$. Therefore

$$
\int_{\mathfrak{E}} f
$$

does not exist at any point in $\mathfrak{B}$ and

cannot then exist.

$$
\int_{\mathbb{B}} \int_{\mathbb{e}} f
$$

The University of ILlinoIs. 